\title{
Sex and SUVmax: Sex-Dependent Prognostication in Early Non-Small Cell Lung Cancer
}

\author{
Zoe Wainer ${ }^{1-3}$, Marissa G. Daniels² ${ }^{2}$ Jason Callahan ${ }^{4}$, David Binns ${ }^{4}$, Rodney J. Hicks ${ }^{4,5}$, Phillip Antippa ${ }^{3,6}$, \\ Prudence A. Russell ${ }^{7}$, Naveed Z. Alam ${ }^{2}$, Matthew Conron ${ }^{8}$, Benjamin Solomon*9, and Gavin M. Wright*1-3 \\ ${ }^{1}$ Department of Surgery, St. Vincent's Hospital, University of Melbourne, Melbourne, Australia; ${ }^{2}$ Department of Cardiothoracic \\ Surgery, St. Vincent's Hospital, Melbourne, Australia; ${ }^{3}$ Division of Surgical Oncology, Peter MacCallum Cancer Centre, Melbourne, \\ Australia; ${ }^{4}$ Centre for Cancer Imaging, Peter MacCallum Cancer Centre, Melbourne, Australia; ${ }^{5}$ Department of Medicine, University \\ of Melbourne, Melbourne, Australia; ${ }^{6}$ Department of Cardiothoracic Surgery, Royal Melbourne Hospital, Melbourne, Australia; \\ ${ }^{7}$ Department of Anatomical Pathology, St. Vincent's Hospital, Melbourne, Australia; ${ }^{8}$ Department of Respiratory Medicine and Sleep \\ Medicine, St. Vincent's Hospital, Melbourne, Australia; and ${ }^{9}$ Department of Haematology and Medical Oncology, Peter MacCallum \\ Cancer Centre, Melbourne, Australia
}

The identification of robust prognostic factors for patients with early-stage non-small cell lung cancer (NSCLC) is clinically important. The International Association for the Study of Lung Cancer has identified both sex and the maximum standardized uptake value (SUVmax) of ${ }^{18} \mathrm{~F}-\mathrm{FDG}$ in the primary tumor as measured by PET as potential prognostic variables. We examined the prognostic value of SUVmax in a surgical cohort of patients with NSCLC and disaggregated the findings by sex. Methods: Patients who had undergone a preoperative PET/CT scan and surgical resection with curative intent from 2001 to 2009 were identified from a prospective database. An SUVmax cutoff was calculated using receiver-operating-characteristic curves. Overall survival was correlated with SUVmax for the whole cohort and disaggregated by sex. Results: Inclusion criteria were met by 189 patients: 127 (67\%) men and 62 (33\%) women. Five-year survival was $54.6 \%$ for the whole cohort, $47.7 \%$ for men, and $68.2 \%$ for women. SUVmax correlated negatively with survival in a univariate analysis for the whole cohort (hazard ratio [HR], 2.51; 95\% confidence interval [Cl], 1.54-4.09; $P<0.001$ ) and men (HR, 3.42; 95\% Cl, 1.94-6.05; $P<0.001$ ) but not for women (HR, 1.61; 95\% Cl, 0.43-3.12; $P=0.77$ ), using 8 as a cutoff. In multivariate analysis, SUVmax correlated with overall survival for the whole cohort $(\mathrm{HR}, 1.70 ; 95 \% \mathrm{Cl}, 1.05-2.99 ; P=0.05)$ and men (HR, 2.40; 95\% Cl, 1.32-4.37; $P=0.004$ ) but not for women (HR, 0.80; 95\% Cl, 0.15-4.47; $P=0.80$ ). Conclusion: SUVmax independently predicted overall survival for men but not for women in this surgical cohort. Our results suggest that SUVmax is an independent prognostic variable in men with surgically treated early NSCLC.

Key Words: ${ }^{18}$ F-FDG PET; prognostic factor; gender

J Nucl Med 2012; 53:1676-1685

DOI: 10.2967/jnumed.112.105197

\footnotetext{
Received Feb. 29, 2012; revision accepted May 30, 2012.

For correspondence or reprints contact: Zoe Wainer, East Melbourne Heart and Lung, 55 Victoria Parade, Fitzroy, Victoria 3065 Australia.

E-mail: zoewainer@gmail.com

${ }^{*}$ Contributed equally to this work.

Published online Sep. 14, 2012.

COPYRIGHT @ 2012 by the Society of Nuclear Medicine and Molecular Imaging, Inc.
}

A ccurate prognostication for patients with non-small cell lung cancer (NSCLC) is both clinically important and elusive. The only validated predictors of tumor behavior and patient outcome are the Tumor Node and Metastasis (TNM) staging system and performance status of the Union Internationale Contre le Cancer/American Joint Cancer Committee (1). This combination lacks sensitivity, and as a result 5-y survival for patients selected for surgery with curative intent is approximately $50 \%$ (2). The International Association for the Staging of Lung Cancer (IASLC) has identified potential prognostic factors including the maximum standardized uptake value (SUVmax) of ${ }^{18} \mathrm{~F}-\mathrm{FDG}$ in the primary tumor as measured by PET and patient sex (1).

In patients with NSCLC, ${ }^{18} \mathrm{~F}-\mathrm{FDG}$ PET is part of the standard of care for mediastinal staging and detection of distant metastasis (3). PET with ${ }^{18} \mathrm{~F}-\mathrm{FDG}$, a radiolabeled form of glucose, exploits genomic and gene alterations in malignant cells that results in alterations in glucose metabolism, an effect first described by Otto Warburg in 1924 (4). There is evidence that alterations in glucose metabolism in primary lung tumors, resulting in greater glucose utilization and hence ${ }^{18}$ F-FDG uptake, may correlate with a more aggressive phenotype $(5,6)$.

The SUVmax of ${ }^{18} \mathrm{~F}$-FDG PET is a semiquantitative measurement of the point of theoretic maximum metabolic rate in a tumor. Many studies have been undertaken to investigate the prognostic potential of SUVmax. A metaanalysis published in 2008, updated in 2010, by Paesmans et al. concluded that SUVmax has a prognostic role in NSCLC (5). However, this metaanalysis was unable to demonstrate that standardized uptake value (SUV) is an independent prognostic factor. Hence, the role of SUVmax in clinical practice remains uncertain.

The World Health Organization reports that global trends in lung cancer incidence demonstrate an increasing burden of disease in women (7) but consistently lower mortality rates $(8)$. The reasons for the lower mortality in women are 
TABLE 1

Baseline Characteristics, Treatment, and Outcome

\begin{tabular}{|c|c|c|c|c|}
\hline Characteristic & All patients & Men (\%) & Women (\%) & $P$ \\
\hline \multicolumn{5}{|l|}{ Patient features } \\
\hline $\operatorname{Sex}(n)$ & & $127(67.2)$ & $62(32.8)$ & Not applicable \\
\hline Age $(y)$ & & & & 0.933321 \\
\hline Median & 70 & 69 & 70 & \\
\hline Range & $43-85$ & $43-85$ & $45-84$ & \\
\hline Ethnicity $(n)$ & & & & 0.7524 \\
\hline Asian descent & $11(5.8)$ & $7(5.5)$ & $4(6.5)$ & \\
\hline Non-Asian & $178(94.2)$ & $120(94.5)$ & $58(93.5)$ & \\
\hline Smoking status $(n)$ & & & & 0.0018 \\
\hline Never smoker & $17(9.2)$ & $5(4)$ & $12(19.4)$ & \\
\hline Ever smoker & $172(90.8)$ & $122(96)$ & $50(80.6)$ & \\
\hline $\mathrm{ECOG}(n)$ & & & & 0.7525 \\
\hline 0 & $116(61.4)$ & $79(62.2)$ & $37(59.7)$ & \\
\hline$\geq 1$ & $73(38.6)$ & $48(37.8)$ & $25(40.3)$ & \\
\hline Diabetes & & & & 0.2145 \\
\hline Present & $32(16.9)$ & 25 (19.7) & 7 (11.3) & \\
\hline Absent & $157(83.1)$ & 102 (62.2) & $55(88.7)$ & \\
\hline \multicolumn{5}{|l|}{ Tumor features $(n)$} \\
\hline Histology & & & & 0.546806 \\
\hline Adenocarcinoma & $99(52.3)$ & $63(49.6)$ & $36(58.1)$ & \\
\hline Squamous cell & $65(34.4)$ & $46(36.2)$ & $19(30.6)$ & \\
\hline Other & 25 (13.3) & $18(14.2)$ & 7 (11.3) & \\
\hline Tumor grade & & & & 0.003933 \\
\hline Poor & $99(53.4)$ & $72(56.7)$ & $27(43.5)$ & \\
\hline Moderate & $78(41.3)$ & $52(40.9)$ & $26(41.9)$ & \\
\hline Well & $12(5.3)$ & $3(2.4)$ & 9 (13.5) & \\
\hline Vascular invasion $(n)$ & & & & 0.354351 \\
\hline Absent & $113(59.7)$ & $73(57.5)$ & $40(64.5)$ & \\
\hline Present & $76(40.3)$ & $54(42.5)$ & $22(35.5)$ & \\
\hline Lymphatic invasion $(n)$ & & & & 0.496035 \\
\hline Absent & $131(69.3)$ & $86(67.7)$ & $45(72.6)$ & \\
\hline Present & $58(30.7)$ & $41(32.3)$ & $17(27.4)$ & \\
\hline Neural invasion $(n)$ & & & & 0.754813 \\
\hline Absent & $172(91)$ & $115(90.5)$ & 57 (91.9) & \\
\hline Present & $17(9)$ & $12(9.5)$ & $5(8.1)$ & \\
\hline Pathologic TNM stage (n) (7th ed.) & & & & $0.042^{*}$ \\
\hline la & $55(29.1)$ & $30(23.6)$ & $25(40.3)$ & \\
\hline $\mathrm{lb}$ & $44(23.3)$ & $30(23.6)$ & $14(22.6)$ & \\
\hline II & $56(29.6)$ & $42(33.1)$ & $14(22.6)$ & \\
\hline III & $26(13.7)$ & $19(14.5)$ & 7 (11.3) & \\
\hline IV & $8(4.3)$ & $6(5.2)$ & $2(3.2)$ & \\
\hline \multicolumn{5}{|l|}{ Treatment details $(n)$} \\
\hline Adjuvant treatment & & & & 0.023804 \\
\hline Yes & $67(35.5)$ & $52(40.9)$ & $15(24.2)$ & \\
\hline No & $122(64.5)$ & $75(59.1)$ & $47(75.8)$ & \\
\hline Surgical approach & & & & 0.656634 \\
\hline Open & $111(58.7)$ & $76(59.8)$ & $35(56.5)$ & \\
\hline Thoracoscopy & 78 (41.3) & $51(40.2)$ & $27(43.5)$ & \\
\hline Resection & & & & 0.604545 \\
\hline Pneumonectomy & $16(8.5)$ & $12(9.4)$ & $4(6.4)$ & \\
\hline Lobectomy & $141(74.6)$ & $96(75.6)$ & $45(72.6)$ & \\
\hline Other anatomic & $15(7.9)$ & $8(6.3)$ & 7 (11.3) & \\
\hline Wedge resection & $17(9)$ & $11(8.7)$ & $6(9.7)$ & \\
\hline Resection type & & & & 0.063776 \\
\hline Anatomic & $172(91.0)$ & $119(93.7)$ & $53(85.4)$ & \\
\hline Nonanatomic & $17(9)$ & $8(6.3)$ & 9 (14.6) & \\
\hline
\end{tabular}




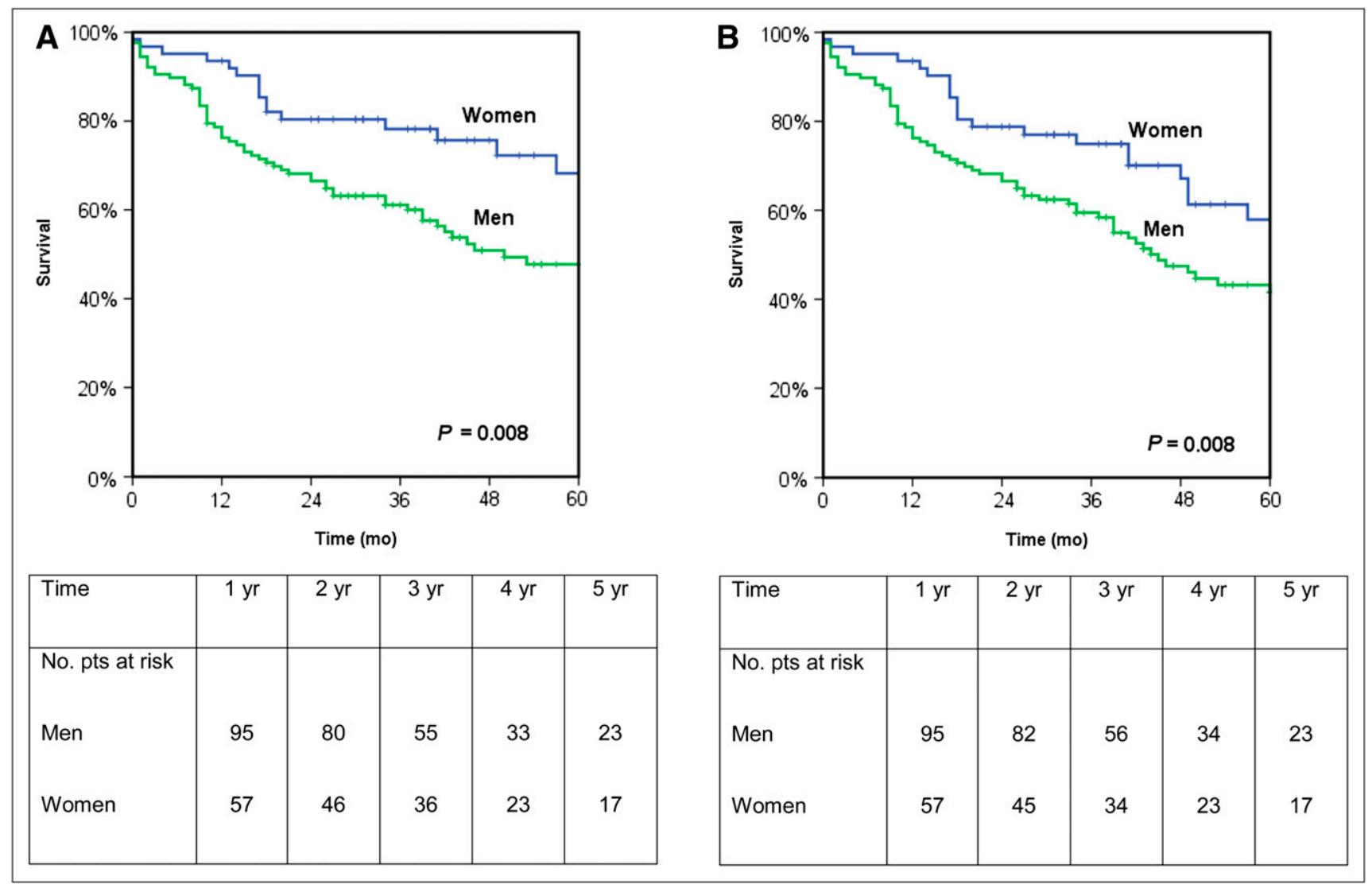

FIGURE 1. (A) Overall 5-y survival. (B) Disease-free 5-y survival. pts = patients.

not well understood but may include important differences in tumor biology. The Lung Cancer Staging Project conducted by the IASLC reported that men, compared with women, have a 1.32 times increased risk of death from NSCLC (9).

Sex and gender are not interchangeable terms, despite this common nomenclature confusion in scientific literature. This study investigated the impact of sex-as defined by the World Health Organization, United Nations, and the American Medical Association Manual of Style $(10,11)$ on the prognostic value of SUVmax.

We conducted a retrospective analysis of SUVmax and patient survival, disaggregated by sex, in a surgical cohort of patients who underwent preoperative PET/CT scans. We hypothesized that SUVmax could stratify patient survival, providing information additional to TNM stage, and sought to examine whether the clinical utility of SUVmax varied by sex.

\section{MATERIALS AND METHODS}

A retrospective analysis of a prospectively maintained surgical database was undertaken. Data collection was performed masked to outcome at the time of SUV calculation and to SUV at the time of survival data analysis. Ethics approval was obtained from the Human Research Ethics Committee at the Peter MacCallum Cancer Centre, and we strictly adhered to the ethics obtained for this research from the committee.
All patients who had undergone resection of histologically confirmed NSCLC between 2001 and 2009 were included if a PET/CT scan had been obtained no longer than 4 wk before surgery performed with curative intent. Those with tumors smaller than the spatial resolution of the PET scanner $(\sim 8 \mathrm{~mm})$ and those who had received neoadjuvant therapy were excluded.

\section{Clinical Data}

The surgical database was supplemented with electronic and hard-copy clinical records to obtain the required patient information relating to demographics, clinical presentation, medical history, staging, diagnosis, treatment, and follow-up.

Patient sex, age at the time of surgery, ethnicity, smoking status, performance status as indicated by Eastern Cooperative Oncology Group (ECOG) score, and the presence of diabetes were noted. Specific staging information obtained included both CT thorax and systematic mediastinal lymph node staging incorporating PET, endobronchial ultrasound-guided fine-needle aspiration, mediastinoscopy, esophageal ultrasound, or comprehensive mediastinal lymph node dissection in line with best clinical practice.

All treatment modalities undertaken as part of therapy with curative intent were documented. The date and extent of surgical therapy were supplemented with details of adjuvant therapy commenced. The location and size of the primary tumor were documented, along with the presence of additional nodules in the resection specimen. Histopathologic examination was performed by a pathologist who reviewed all original slides to determine the pathologic classification of tumors and tumor grade according to the 2004 World Health Organization 


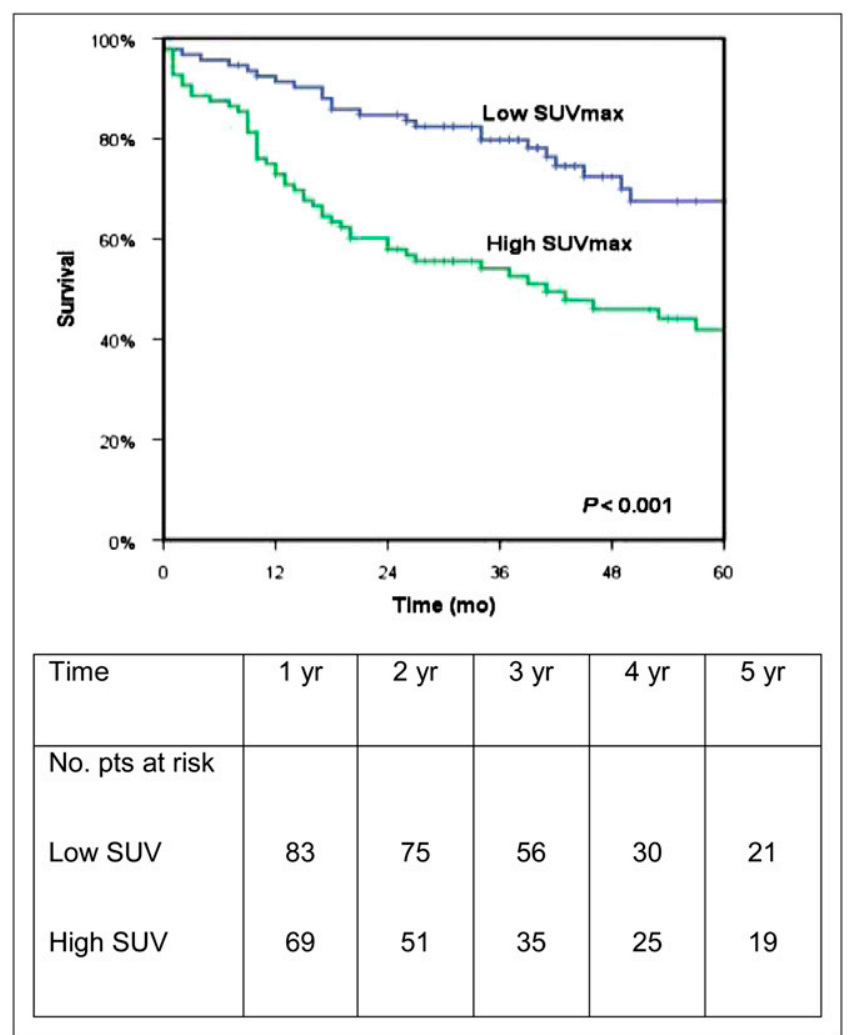

FIGURE 2. Data showing correlation between SUVmax and overall survival. pts $=$ patients.

definition $(12,13)$ and to determine the presence of visceral or parietal pleural, vascular, lymphatic, and neural invasion. Pathologic TNM stage was then determined according to the seventh TNM revision (14). Follow-up data consisted of date of last contact and status at this time and the date of first recurrence and date of death if required.

\section{${ }^{18}$ F-FDG PET Scan}

All patients underwent combined PET/CT on a Discovery LS or Discovery STE (GE Healthcare). Patients fasted for at least $6 \mathrm{~h}$ before PET. A PET/CT scan was acquired from the base of skull to proximal thighs approximately $60 \mathrm{~min}$ after radiotracer injection. Patient plasma glucose level was measured before injection, and PET was performed only if this was less than $10 \mathrm{mmol} / \mathrm{L}$. The scan was processed using ordered-subset expectation maximization iterative reconstruction with attenuation correction. An in-house program (MARVn 2.16) was used to contour the primary lesion and calculate the SUV. A semiautomatic autocontouring process with an SUV threshold of 2.5 was used to define the volume of interest. The threshold value could be changed at the operator's discretion in order to contour the primary lesion in areas deemed likely to represent inflammation in the peripheral lung. First, a 3-dimensional-search region of interest was drawn around visible tumor in a suitable reference transaxial plane, excluding myocardium and other avid nonmalignant structures. The volume of interest was then defined by adapting an orthogonal region of interest on the sagittal plane to encompass the craniocaudal extent of the primary and on the coronal plane, ensuring that the mediolateral extent of tumor was covered; correlative CT thorax was used to delineate between areas of atelectasis or obstructive pneumonitis and tumor.

\section{SUV Calculation}

All PET scans were first interpreted clinically by a nuclear medicine physician and then were reevaluated for the calculation of SUVmax for the purposes of this study, with SUVmax calculated per the formula in Paquet et al. (15). SUVmax, mean SUV (SUVmean), SUVmax lean body mass, and tumor volumes were calculated for the primary lesion in all patients. SUVmax was defined as the single pixel of maximum value, and SUVmean

TABLE 2

Correlation Between SUVmax and Overall Survival

\begin{tabular}{|c|c|c|c|c|c|c|}
\hline \multirow[b]{2}{*}{ Factor } & \multicolumn{2}{|c|}{ Whole cohort } & \multicolumn{2}{|l|}{ Men } & \multicolumn{2}{|c|}{ Women } \\
\hline & $\mathrm{HR}$ & $P$ & $\mathrm{HR}$ & $P$ & $\mathrm{HR}$ & $P$ \\
\hline \multicolumn{7}{|l|}{ Pairwise analysis* } \\
\hline Age & 2.53 (1.55-4.12) & $<0.001$ & 3.43 (1.93-6.01) & $<0.001$ & $1.31(0.48-3.54)$ & 0.600 \\
\hline Sex & $2.71(1.66-4.42)$ & $<0.001$ & Not applicable & & Not applicable & \\
\hline ECOG & $2.30(1.41-3.66)$ & 0.001 & 3.08 (1.73-5.47) & $<0.002$ & $1.13(0.415-3.06)$ & 0.816 \\
\hline Smoking & $2.53(1.54-4.16)$ & $<0.001$ & $3.46(1.96-6.12)$ & $<0.001$ & $1.14(0.40-3.28)$ & 0.807 \\
\hline Histology & 2.32 (1.42-3.78) & 0.001 & $2.96(1.65-5.30)$ & $<0.001$ & $1.17(0.43-3.15)$ & 0.757 \\
\hline Tumor grade & 2.27 (1.37-3.75) & 0.001 & $3.25(1.82-5.81)$ & $<0.001$ & $0.94(0.31-2.76)$ & 0.908 \\
\hline Pathologic TNM stage & 2.031 (1.22-3.38) & 0.006 & $2.96(1.58-5.56)$ & 0.001 & $1.05(0.39-2.83)$ & 0.928 \\
\hline Diameter & 2.28 (1.34-3.87) & 0.002 & $3.9(1.81-6.33)$ & $<0.001$ & $1.06(0.36-3.09)$ & 0.912 \\
\hline Vascular invasion & $2.27(1.38-3.74)$ & 0.001 & $3.21(1.81-5.72)$ & $<0.001$ & $0.92(0.33-2.56)$ & 0.866 \\
\hline Lymphatic invasion & $2.24(1.36-3.68)$ & 0.002 & $3.27(1.83-5.85)$ & $<0.001$ & $0.85(0.31-2.34)$ & 0.749 \\
\hline Neural invasion & $2.43(1.49-3.96)$ & $<0.001$ & 3.37 (1.91-5.96) & $<0.001$ & $0.99(0.36-2.73)$ & 0.99 \\
\hline Diabetes & 2.51 (1.54-4.09) & $<0.001$ & 3.44 (1.94-6.07) & $<0.001$ & 1.05 (0.38-2.87) & 0.932 \\
\hline Multivariate analysis ${ }^{\dagger}$ & $1.70(1.05-2.99)$ & 0.050 & $2.40(1.32-4.37)$ & 0.004 & $0.80(0.15-4.47)$ & 0.800 \\
\hline
\end{tabular}

${ }^{*}$ Correlation between SUVmax and survival, controlling for prognostic factors.

${ }^{\dagger}$ Correlation between SUVmax and survival, controlling for all prognostic factors.

Data in parentheses are $95 \% \mathrm{Cls}$. 


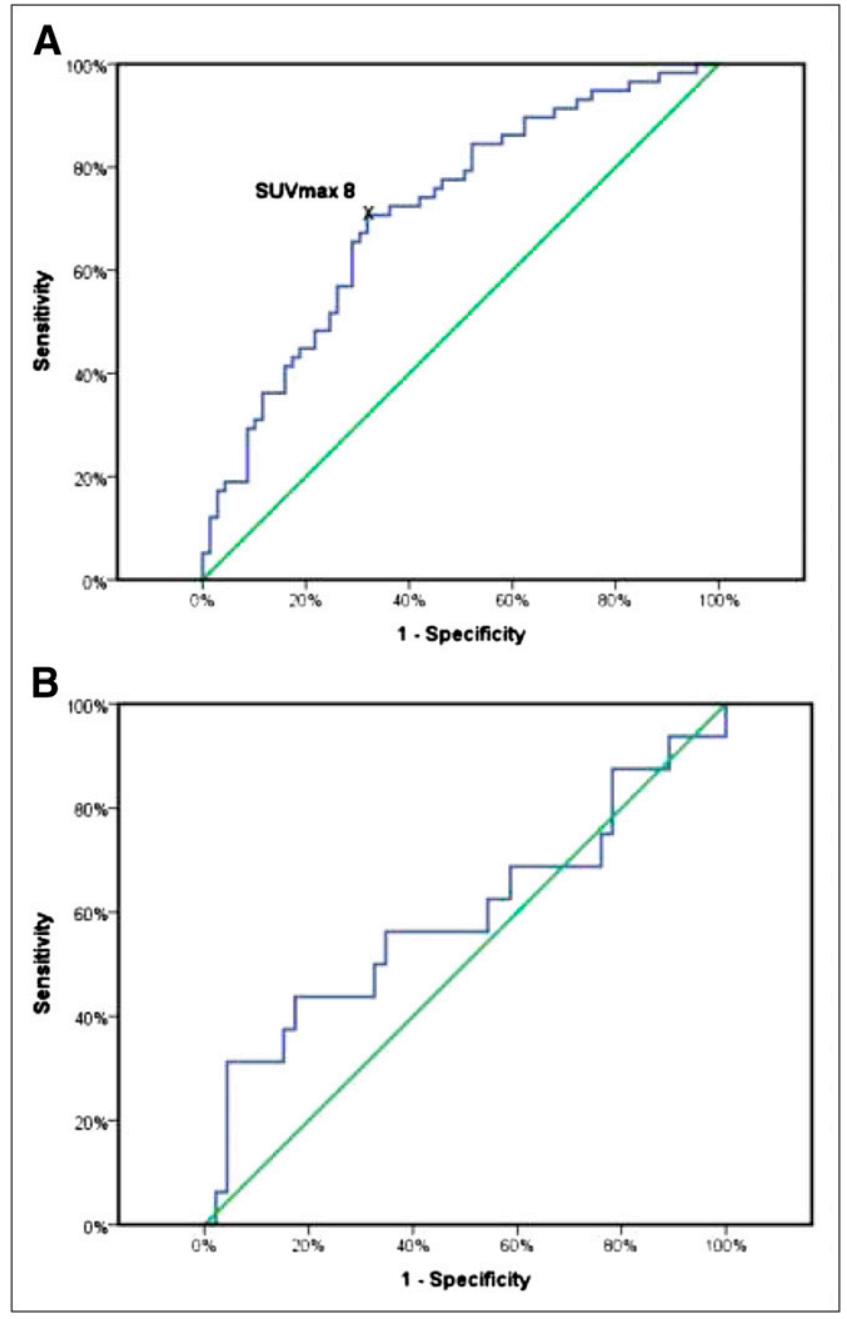

FIGURE 3. ROC curves for SUVmax and overall survival in men (area under curve, $0.715 ; P<0.001 ; 95 \% \mathrm{Cl}, 0.626-0.804)(\mathrm{A})$ and in women (area under curve, 0.591; $P=0.281 ; 95 \% \mathrm{Cl}, 0.411-0.771$ ) (B).

represents the mean pixel value in the volume of interest. The liver SUVmean was also recorded.

\section{Lean Body Mass SUVmax}

SUVmax mathematically adapted to lean body mass was calculated according to a published and validated formula (15).

\section{Liver SUVmean}

Liver SUVmean was calculated, per the methodology used for tumor SUVmean, at the time of PET/CT acquisition for quality control, providing a means to control for any errors in camera calibration factors or operator-entered parameters required for accurate SUV determination (16). If identified as being outside our established range, acquisition and patient-related parameters were verified and corrected where necessary. No patients were excluded from this study on that basis.

\section{Statistical Analysis}

The primary endpoint was all-cause mortality calculated from the time of pathologic diagnosis to the date of last contact or death. Recurrence was pathologically confirmed, with unequivocal progression of radiologic findings considered an acceptable surrogate. Patient and tumor characteristics were expressed as frequencies with mean and SD or median and range. Comparisons were made using the $\chi^{2}$ test, Fisher exact test, and the independent-samples median test.

SUV was obtained and survival analyses were performed on the whole cohort and repeated with data disaggregated by sex. Univariate and multivariate analyses were performed using Kaplan-Meier survival curves, the log-rank test, and the Cox proportional-hazards model. Diabetes and lymphatic, vascular, and neural invasion, in addition to the prognostic factors described in the IASLC staging manual (1)—specifically pathologic stage, ECOG performance status, ethnicity, age, sex, histology, tumor grade, and diameter-were examined for association with survival. The pattern of distribution of SUV and the nature of its association with survival were examined with locally weighted scatterplot smoothing, Spearman $\rho$ correlation, and Cox regression.

Three methods were used to identify an SUV cutoff predictive of outcome: median, receiver-operating-characteristic (ROC) curve, and log-rank selection of best cutoff. The prognostic effect of SUV cutoff was quantified as a hazard ratio (HR) with $95 \%$ confidence interval (CI) and a $P$ significance level of 0.05 or less. The accuracy of SUVmax to predict 3-y survival was expressed as positive and negative predictive value and positive and negative likelihood ratio. The probability of test accuracy was determined using Bayes theorem. All analyses were repeated using lean body mass SUVmax.

\section{RESULTS}

One hundred eighty-nine patients were included in the analysis: $127(67 \%)$ men and $62(33 \%)$ women (Table 1). Median age was $70 \mathrm{y}$, and median follow-up was $35 \mathrm{mo}$, with a range of 3-109 mo. Five-year outcome data were available for $116(61 \%)$ patients. A statistically significant difference between men and women was found in that men were more likely to have a smoking history, poorly differentiated tumors, and higher pathologic TNM stage. There was no statistically significant difference between men and women with respect to histology type, ECOG score, diabetes, or ethnicity. Adjuvant chemotherapy administration differed in line with TNM staging (Table 1). Liver SUVmean was consistent with published data (15).

\section{Survival}

Overall 5-y survival for the whole cohort was 54.6\% and was statistically significantly greater for women $(68.2 \%)$ than for men $(47.7 \%)(P=0.003$, Fig. 1A). The HR for men was 2.19 (95\% CI, 1.28-3.75; $P=0.004)$. Median survival was 34 mo overall and 33 and 40 mo for men and women, respectively. Survival differences between men and women persisted after multivariate analysis (HR, 2.36; 95\% CI, 1.31-4.26; $P=0.004)$.

Disease-free 5-y survival was $46.9 \%$ for the whole cohort, $57.9 \%$ for women, and $41.6 \%$ for men $(P=0.008$, Fig. 1B). The effect persisted in multivariate analysis, with men at greater risk of disease recurrence than women (HR, 2.24; 95\% CI, 1.31-3.83; $P=0.003)$.

\section{Correlation Between SUV and Overall Survival}

The association between SUVmax, SUVmean, and overall survival was not linear. Because SUVmean and SUVmax 


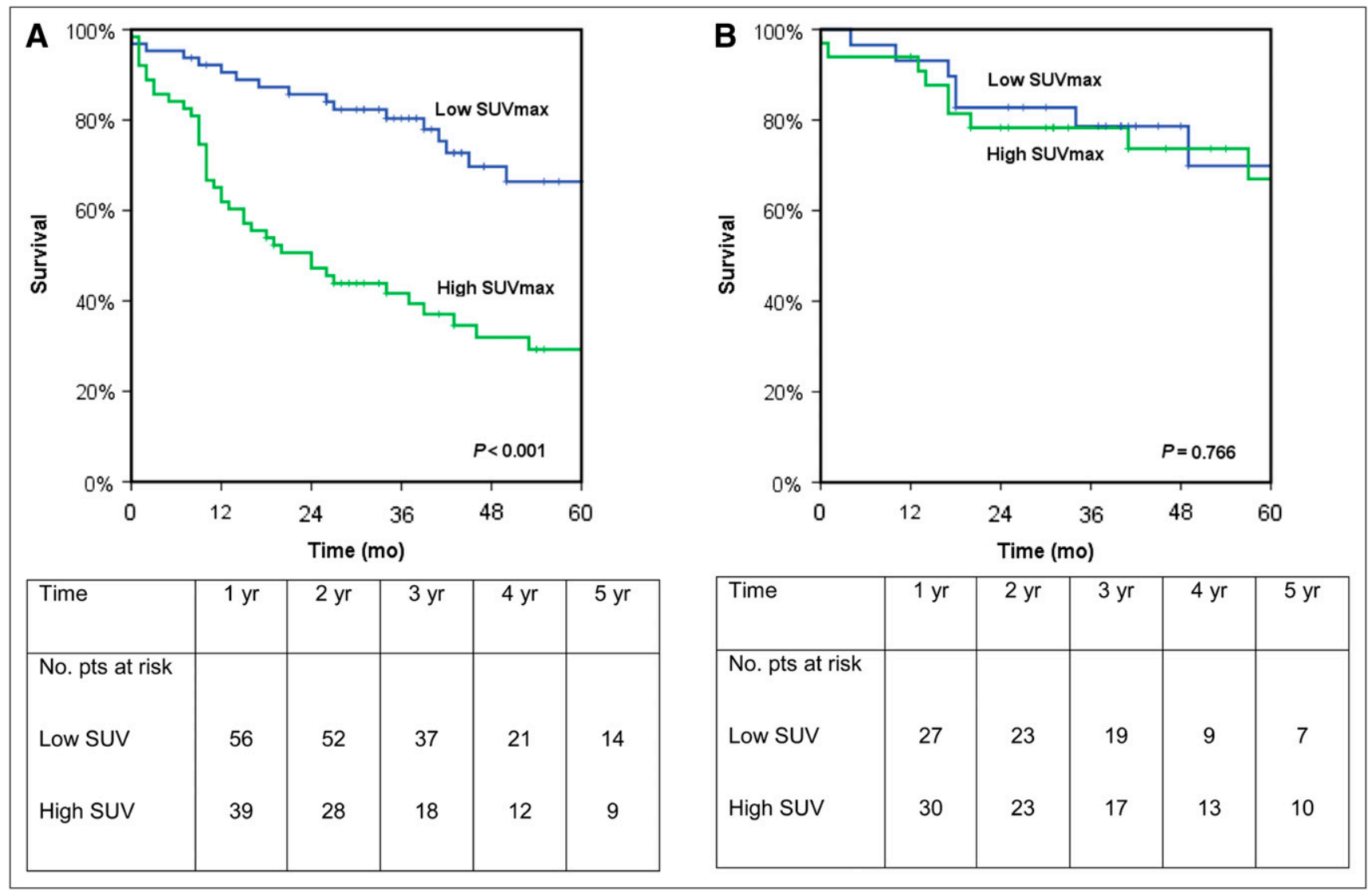

FIGURE 4. Data showing correlation between SUVmax and overall survival in men (A) but not in women (B). pts = patients.

are codependent variables with a similar distribution and nature of association with survival, and because of the advantageous operator-independent calculation of the latter, the SUVmax cutoff was selected as the prognostic factor for evaluation in this study.

The optimal ROC curve SUVmax cutoff of 8 was consistent with both median and log-rank selection methods and was used for all subsequent analyses because it takes into account the sensitivity and specificity of the cutoff point. An SUVmax below this cutoff occurred in 93 (49.2\%) patients, with 96 (50.8\%) having an SUVmax above this value.

In the whole cohort, SUVmax correlated significantly with overall survival in univariate analysis (HR, 2.51; 95\% CI, 1.54-4.09, $P<0.001$; Spearman $\rho,-0.269, P<0.000$; Fig. 2) and multivariate analysis (HR, 1.70; 95\% CI, 1.002.99, $P=0.05$; Table 2).

\section{Correlation Between SUV and Survival for Men and Women}

The distribution of SUVmax as a continuous variable was similar for men and women. Sixty-three men (49.6\%) and 33 women $(53.2 \%)$ had an SUVmax of 8 or more. Delineation of SUVmax quartiles demonstrated a comparable distribution for the 25th, 50th, and 75th quartiles: 4.41, 7.95 , and 13.21 for men, respectively, and 5.08, 8.44, and 13.54 for women, respectively.
Men had an SUVmax cutoff of 8.0 using an ROC curve (Fig. 3A), consistent with median and log rank selection of best cutoff. No SUVmax ROC curve cutoff was identifiable for women (Fig. 3B). The median SUVmax in women, 8.43, did not correlate with survival. An SUVmax cutoff of 17 was identified using the log-rank method. Because the prognostic value of this was lost in multivariate analysis and only $8(13 \%)$ women had tumors with an SUVmax of 17 or more, the same SUVmax cutoff of 8 was used in men and women for clinical applicability.

In univariate analysis, SUVmax correlated significantly with overall survival for men (HR, 3.42; 95\% CI, 1.946.05; $P<0.001$ ) but not for women (HR, 1.16; 95\% CI, $0.43-3.12, P=0.77$; Figs. $4 \mathrm{~A}$ and $4 \mathrm{~B})$. The Spearman $\rho$ correlation coefficient was -0.39 for men $(P<0.001)$ and 0.036 for women $(P=0.78)$. For men, SUVmax cutoff retained its prognostic value in multivariate analysis (HR, 2.40; 95\% CI, 1.32-4.37; $P=0.004$; Table 2).

\section{Disease-Free Survival}

The ROC curve cutoff was 8 (Fig. 5A), consistent with the median, 8.06, and log-rank selection of best cutoff, for both the whole cohort and men. No ROC curve cutoff was identified in women (Fig. 5B). In univariate analysis, SUVmax correlated with disease-free survival for the whole cohort (HR, 1.81; 95\% CI, 1.20-2.74; $P=0.005$ ) and in men (HR, 


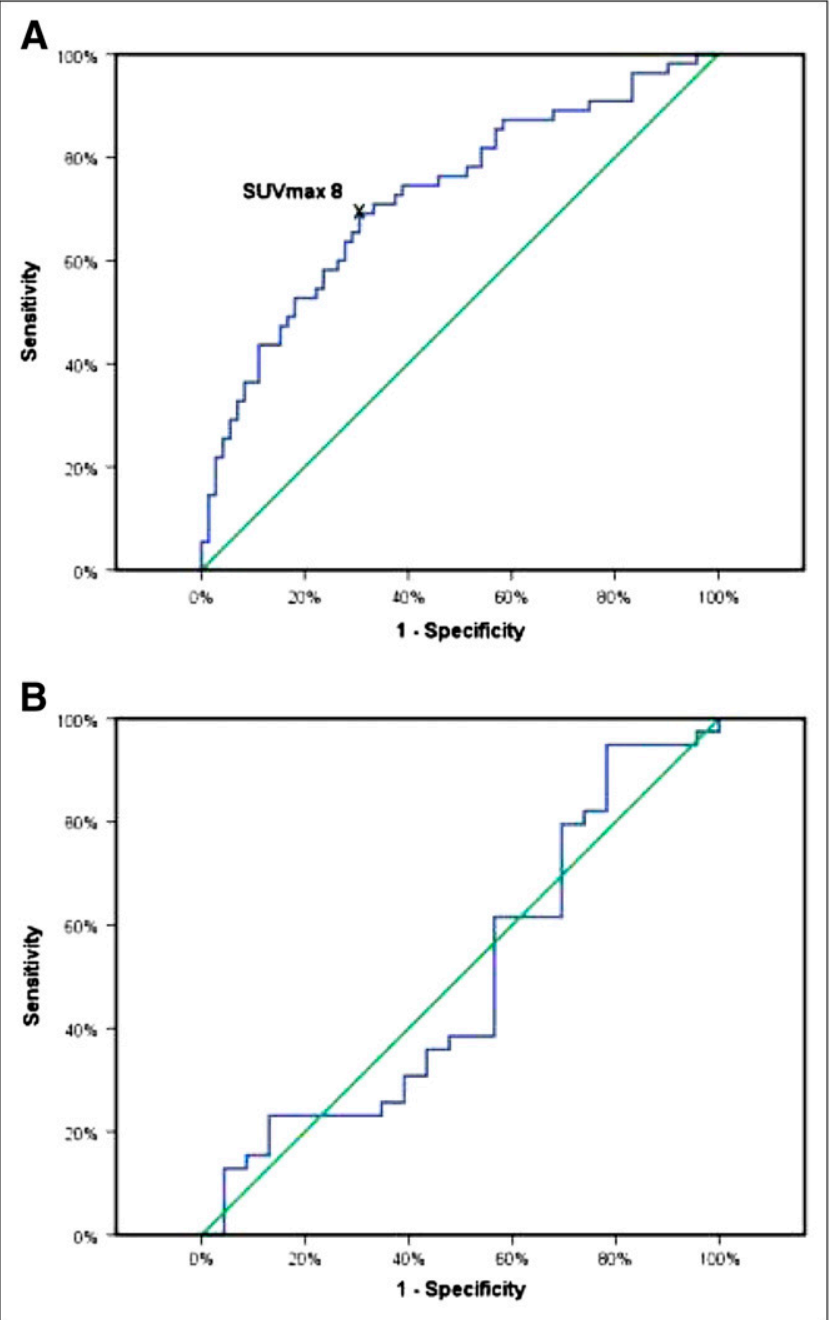

FIGURE 5. ROC curves for SUVmax and disease-free survival in men (area under curve, 0.727; $P<0.001 ; 95 \% \mathrm{Cl}, 0.637-0.816)(\mathrm{A})$ and in women (area under curve, 0.494; $P=0.946$; 95\% Cl, 0.338-0.649) (B).

2.77; 95\% CI, 1.70-4.51; $P<0.001$ ) but not women (HR, 0.67 ; 95\% CI, 0.29-1.53; $P=0.34$; Figs. 6A and 6B). In multivariate analysis, an SUVmax of 8 was not prognostic for women but was for the entire cohort and for men (HR, 2.29 ; 95\% CI, 1.26-4.16, $P=0.007)$. Differences between men and women were statistically significant $(P<0.001$, Table 3).

\section{Lean Body Mass SUVmax}

Lean body mass SUVmax data were available for 147 PET scans (101 [69\%] men and 46 [31\%] women). Baseline characteristics were not significantly different between the overall cohort and the lean body mass SUVmax subcohort. The SUVmax cutoff was 8 for the overall cohort and for men, but no overall cutoff could be identified for women. The SUVmax cutoff was predictive of survival for men in multivariate analysis but not for women. Results between the lean body mass SUVmax and overall cohort were equivalent (supplemental materials are available online only at http:// jnm.snmjournals.org).

\section{DISCUSSION}

The development of the malignant potential of a cell is associated with significant changes in glucose metabolism (17). The observation that cancer cells metabolize glucose largely by glycolysis, rather than oxidative phosphorylation, was first made by Warburg in the early 1900 s $(4,17)$. This process results in tumor cells using glucose beyond their apparent bioenergetic requirements. It has been proposed that altered glucose metabolism in malignant cells reflects tumor behavior, and studies have correlated increased rates of glucose metabolism with increased malignant potential $(18,19)$. A better understanding of tumor glucose metabolism may facilitate an understanding of how SUVmax may be a prognostic tool.

A definitive explanation for the process by which increased glucose metabolism by cancer cells conveys a survival advantage for the tumor remains elusive. Hypoxia-inducible factor 1 (HIF-1), a transcription factor upregulated in cancer cells, coordinates a large number of intracellular processes that are advantageous for tumor cells, including intracellular metabolism of glucose (17). Intratumoral expression of HIF-1 can be constitutively upregulated, for example, by mutations in genes such as the Von Hippel Lindau genes. Thomas et al. have demonstrated an association among Von Hippel Lindau knockout, increased HIF expression, and increased ${ }^{18} \mathrm{~F}-\mathrm{FDG}$ avidity (20). Tissue hypoxia results in further increases in HIF-1 expression, conferring a survival advantage for cells harboring this adaptation and resulting in a more aggressive tumor phenotype (21). These cells must metabolize glucose by glycolysis, rather than oxidative phosphorylation, to prevent apoptosis, thereby increasing their glucose utilization. In turn, high levels of glucose metabolism, as inferred from ${ }^{18}$ F-FDG PET studies, have been shown to correlate with poor outcome in patients with NSCLC $(5,6)$.

A metaanalysis by Paesmans et al. (5), conducted by the European Lung Cancer Working party for the IASLC Lung Cancer Staging Project, analyzed 21 studies that evaluated the prognostic role of SUVmax in NSCLC. This metaanalysis found that patients with a high SUVmax had a shorter survival than patients with a lower SUVmax, with an overall HR of 2.08 (5). Our study supported this conclusion, with an HR of $2.51(P<0.001)$ in a surgical cohort. We also demonstrated a discrepancy between the prognostic value of SUVmax in men and women. After disaggregation of data by sex, the strength of association between a high SUVmax and an aggressive tumor was strengthened in men, with no correlation found for women. The metaanalysis of Paesmans et al. did not examine sex differences in the correlation between SUVmax and survival.

After sex disaggregation of our data, the HR for men with a high SUVmax was $3.42(P<0.001)$, whereas the HR for women was not significant, irrespective of SUVmax cutoff calculation method $(P=0.77)$. Spearman $\rho$ correlation was not significant for the overall cohort or women but demonstrated a correlation between SUVmax and overall survival in men, with a value of $-0.387(P<0.001)$. In 


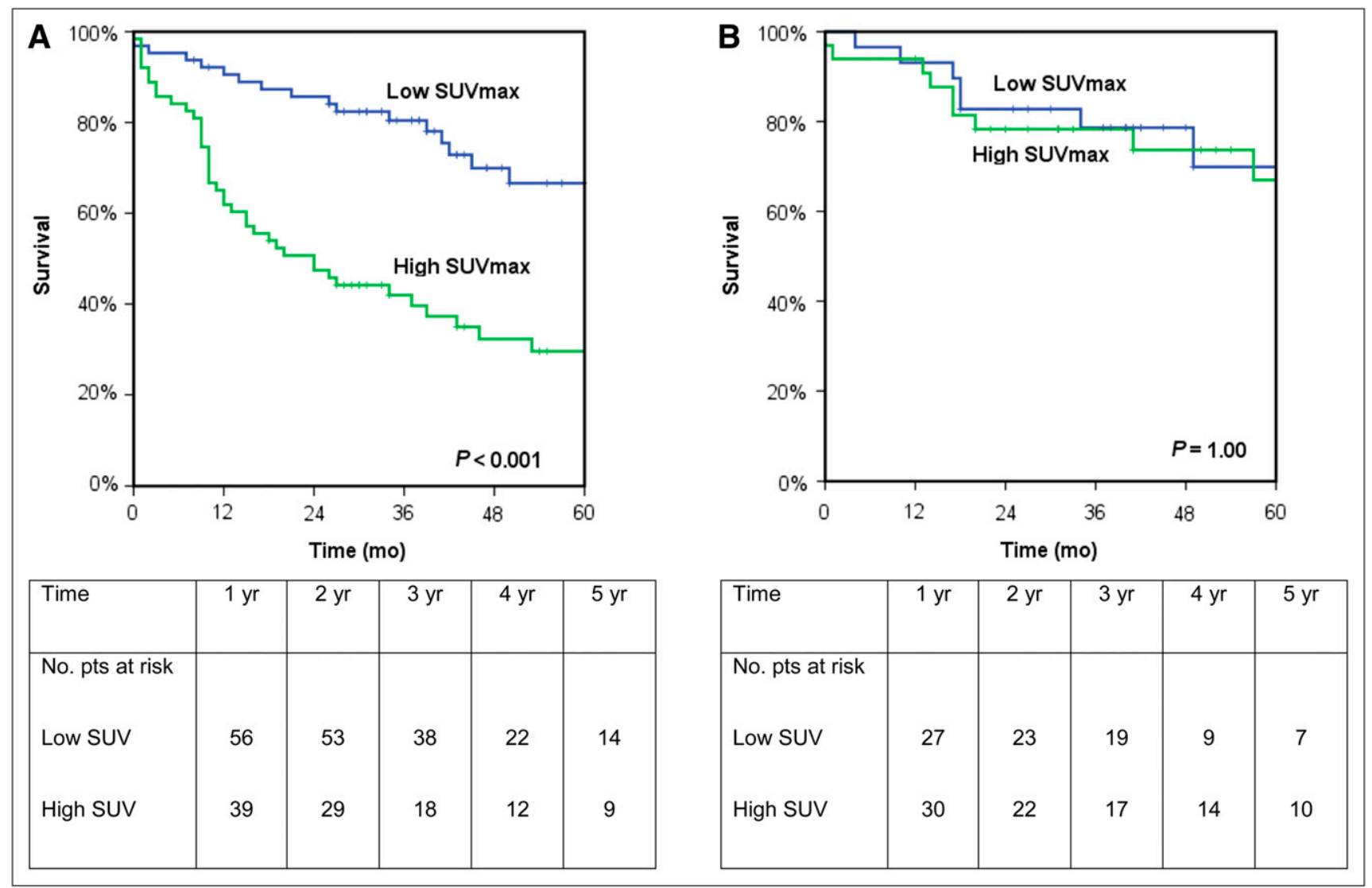

FIGURE 6. Data showing correlation between SUVmax and 5-y disease-free survival in men (A) but not in women (B). pts = patients.

multivariate analysis, the HR for men with a high SUVmax was $2.40(P=0.004)$, whereas the HR for women was not significant. Although there was an imbalance in the proportion of poorly and moderately differentiated tumor grade in men and women (Table 1), this imbalance does not seem to account for the sex differences observed because, on pairwise analysis controlling for tumor grade, the HR for men with a high SUVmax remained statistically significant at $3.35(P<0.001)$. Again no statistical significance was demonstrated in the female cohort.

TABLE 3

Correlation Between SUVmax and Disease-Free Survival

\begin{tabular}{|c|c|c|c|c|c|c|}
\hline \multirow[b]{2}{*}{ Factor } & \multicolumn{2}{|c|}{ Whole cohort } & \multicolumn{2}{|c|}{ Men } & \multicolumn{2}{|c|}{ Women } \\
\hline & $\mathrm{HR}$ & $P$ & $\mathrm{HR}$ & $P$ & $\mathrm{HR}$ & $P$ \\
\hline \multicolumn{7}{|l|}{ Pairwise analysis* } \\
\hline Age & $1.85(1.23-2.80)$ & 0.003 & $2.93(1.79-4.80)$ & $<0.001$ & $0.70(0.31-1.61)$ & 0.401 \\
\hline Sex & $1.99(1.31-3.00)$ & 0.001 & Not applicable & & Not applicable & \\
\hline ECOG & 1.67 (1.10-2.54) & 0.016 & $2.64(1.61-4.32)$ & $<0.001$ & 0.60 (0.26-1.39) & 0.234 \\
\hline Histology & $1.70(1.12-2.57)$ & 0.012 & $2.47(1.49-4.09)$ & $<0.001$ & $0.68(0.30-1.55)$ & 0.357 \\
\hline Grade & $1.64(1.07-2.52)$ & 0.023 & $2.69(1.63-4.44)$ & $<0.001$ & $0.50(0.20-1.26)$ & 0.143 \\
\hline Pathologic TNM stage & $1.42(0.92-2.19)$ & 0.109 & $2.45(1.40-4.26)$ & 0.002 & $0.60(0.26-1.39)$ & 0.233 \\
\hline Diameter & $1.57(1.00-2.47)$ & 0.05 & $2.76(1.60-4.75)$ & $<0.001$ & $0.52(0.21-1.28)$ & 0.155 \\
\hline Vascular invasion & $1.70(1.12-2.59)$ & 0.013 & $2.77(1.69-4.56)$ & $<0.001$ & $0.50(0.21-1.20)$ & 0.119 \\
\hline Lymphatic invasion & $1.65(1.09-2.52)$ & 0.019 & $2.77(1.69-4.55)$ & $<0.001$ & $0.46(0.19-1.09)$ & 0.079 \\
\hline Neural invasion & $1.76(1.16-2.66)$ & 0.008 & $2.78(1.70-4.55)$ & $<0.001$ & $0.58(0.25-1.37)$ & 0.216 \\
\hline Diabetes & $1.84(1.21-2.77)$ & 0.004 & $2.85(1.75-4.66)$ & $<0.001$ & $0.66(0.28-1.55)$ & 0.339 \\
\hline Multivariate analysis $^{\dagger}$ & $1.28(0.79-2.08)$ & 0.318 & $1.96(1.08-3.58)$ & 0.027 & $0.47(0.14-1.56$ & 0.22 \\
\hline
\end{tabular}


For completeness, we examined the impact of lean body mass, an obvious sex difference, in a representative subset of patients and found identical results, indicating that body composition was not a factor in the identified sex differences.

Hoang et al. found no correlation with SUVmax and survival in a cohort of patients with advanced NSCLC (22). They examined differences and distribution of high and low SUVmax in both men and women. They found that women had a survival advantage, and the percentages of men and women with both high and low SUV were similar, as in our study. However, this study examined the correlation of SUVmax and survival only in clinically advanced NSCLC, which represents a different patient population. Similarly, Vesselle et al. found no correlation when examining SUVmax and survival. However, their cohort consisted of $49 \%$ with either stage III or stage IV (23). They examined the subcohort of patients with early NSCLC and again found no correlation in 103 patients. In this study, women constituted $31 \%$ of the cohort with surgically treated early NSCLC, and it is possible that the failure to identify a correlation between SUV and survival was because data were not disaggregated on the basis of sex (23).

Population-based data demonstrate that lung cancer behaves differently in women and men (7). Irrespective of stage, histology, or treatment, women live longer than their male counterparts (24). Data from the Surveillance, Epidemiology and End Results Database (SEER) indicate that the 5-y survival of women is $18.7 \%$, compared with men at $14.1 \%$ (25). This pattern is consistent with our surgical cohort, in which women had a 5-y survival of $68.2 \%$, compared with men at $47.7 \%$. In multivariate analysis, men were 2.36 times more likely to die within $5 \mathrm{y}$ than women $(P=0.004)$. The difference in survival between men and women strongly indicates divergence in tumor biology.

Knowledge of the sex differences in NSCLC biology will enhance understanding of mechanisms of carcinogenesis that will benefit all patients. A 2011 Lancet editorial strongly encouraged researchers to routinely analyze data by sex, noting that sex differences might be a more important factor in health and disease than is currently understood (26). Molecular differences between NSCLC in men and women, such as capacity for DNA repair (27) and rates of driver mutations (28), may contribute to differences in the natural history of NSCLC between the sexes. Epidermal growth factor receptor gene mutations-recognized driver mutations in $8 \%-10 \%$ of NSCLC (29)-and K-ras mutations-present in 10\%-30\% of NSCLC (28) - have both been more commonly observed in women than men.

There is also evidence of sex differences in the natural history of other malignancies. Population-based data have been analyzed disaggregated by sex for several cancers. Cook et al. published an analysis of 36 cancers from the SEER database from 1977 to 2006 and found that for most cancers, age-adjusted mortality rates were higher among men then women (30). SEER data reporting survival for limited-stage small cell lung cancer has shown superior survival among women (31), as have survival data from the Eindhoven Cancer Registry in The Netherlands for cutaneous melanoma and Hodgkin lymphoma (32). Ongoing investigation of possible mechanisms of sex differences in tumor biology is required.

We recognize the limitations of this study. Many factors influence the accuracy of SUV measurement, including patient weight, glycemia, time of injection, lesion movement, and partial-volume effect. We attempted to address these limitations by controlling for diabetes in univariate and multivariate analyses and by considering patient weight as a component of the SUVmax calculation. Most patients presenting for PET at our institution have locally advanced lung cancer that is unlikely to be subject to partial-volume effects; early-stage disease does not appear to have contributed to the improved prognosis for women. As respiratory gating was unavailable, respiratory blurring is a potential confounding factor in assessing the SUV of lung lesions, particularly those in the base of the lung (33). However, there is no reason to suspect that this was a factor in the results obtained. A further limitation is the retrospective nature of the study and its 7-y span, during which time best clinical practice changed. It is unlikely that these changes account for observed differences in outcome between men and women, because there was no treatment bias toward either sex.

These data cannot provide a mechanistic explanation for the lack of correlation between SUVmax and outcome in women. Furthermore, the findings require validation in an independent cohort. Subgroup analyses may proffer an explanation for our findings, but such analyses would be underpowered. Despite low numbers and the pitfalls of subgroup analysis, this is a large SUV series, and the difference found between men and women has important implications for the application of SUVmax as a prognostic factor in surgical cohorts.

\section{CONCLUSION}

SUVmax in our surgical cohort showed a strong correlation with outcome in men but no correlation with outcome in women. We believe these findings suggest that NSCLC in men and women may represent differences in disease processes with distinct prognostic factors, thereby adding to the body of evidence that there is sexual dimorphism in tumor biology. Identification of factors resulting in noncorrelation in women may provide insight into a disease phenotype or risk profile in men for which SUVmax is not prognostic and may provide novel insight into the biology of NSCLC.

\section{DISCLOSURE STATEMENT}

The costs of publication of this article were defrayed in part by the payment of page charges. Therefore, and solely to indicate this fact, this article is hereby marked "advertisement" in accordance with 18 USC section 1734. 


\section{ACKNOWLEDGMENTS}

We acknowledge and thank Richard Williams, David Ball, Dr. Richard Fisher, Dr. Eddie Lau, and Dr. Thomas Zander for their guidance and contribution in the completion of this manuscript. Dr. Zoe Wainer was the recipient of the Royal Australasian College of Surgeons Raelene Boyle Scholarship. No other funding sources were obtained for this research. No other potential conflict of interest relevant to this article was reported.

\section{REFERENCES}

1. Brundage MD, Mackillop WJ. Prognostic factors in thoracic malignancies. In: Goldstraw P, Asamura H, Bunn P, et al., eds. Staging Manual in Thoracic Oncology. Orange Park, FL: Editorial Rx Press; 2009:111-118.

2. Groome PA, Bolejack V, Crowley JJ, et al. The IASLC lung cancer staging project: validation of the proposals for revision of the $\mathrm{T}, \mathrm{N}$, and $\mathrm{M}$ descriptors and consequent stage groupings in the forthcoming (seventh) ed. of the TNM classification of malignant tumours. J Thorac Oncol. 2007;2:694-705.

3. Pieterman RM, Putten JWG, Meuzelaar JJ, et al. Preoperative staging of nonsmall-cell lung cancer with positron-emission tomography. $N$ Engl J Med. 2000;343:254-261.

4. Warburg O. Origin of cancer cells. Science. 1956;123:309-314.

5. Paesmans M, Berghmans T, Dusart M, et al. Primary tumor standardized uptake value measured on fluorodeoxyglucose positron emission tomography is of prognostic value for survival in non-small cell lung cancer update of a systematic review and meta-analysis by the European Lung Cancer Working Party for the International Association for the Study of Lung Cancer Staging Project. J Thorac Oncol. 2010;5:612-619.

6. Dooms C, van Baardwijk A, Verbeken E, et al. Association between ${ }^{18}$ F-fluoro2-deoxy-D-glucose uptake values and tumor vitality: prognostic value of positron emission tomography in early-stage non-small cell lung cancer. J Thorac Oncol. 2009;4:822-828.

7. Ferlay J, Shin HR, Bray F, Forman D, Mathers C, Parkin DM. GLOBOCAN 2008 v1.2, Cancer Incidence, Mortality and Prevalence Worldwide in 2008. Lyon, France: International Agency for Research on Cancer, World Health Organization; 2010. Available at: http://globocan.iarc.fr. Accessed August 13, 2012.

8. Patel JD, Bach PB, Kris MG. Lung cancer in US women: a contemporary epidemic. JAMA. 2004;291:1763-1768.

9. Chansky KM, Sculier JP, Crowley JJ, et al. The International Association for the Study of Lung Cancer Staging Project: prognostic factors and pathologic TNM stage in surgically managed non-small cell lung cancer. J Thorac Oncol. 2009;4:792-801.

10. WHO Department of Gender. Women and Health. Gender in Lung Cancer and Smoking Research. Geneva, Switzerland: World Health Organization; 2004:6.

11. Iverson C, Christiansen S, Flanagin A, eds. AMA Manual of Style. 10th ed. New York, NY: Oxford University Press; 2009; chap 11.10.1. http://www. amamanualofstyle.com/oso/private/content/jama/9780195176339/p110.html?q=sex| gender\#jama-9780195176339-div2-350. Accessed April 30, 2012.

12. Travis WD, Brambilla E, Muller-Hermelink HK, Harris CC. World Health Organisation classification of tumors: pathology and genetics of tumors of the lung, pleura, thymus and heart. Lyon, France: IARC Press; 2004:26-31
13. Travis WD, Brambilla E, Muller-Hermelink HK, Harris CC. World Health Organisation classification of tumors: pathology and genetics of tumors of the lung, pleura, thymus and heart. Lyon, France: IARC Press; 2004:35-51.

14. Lung. In: Edge SB, Byrd DR, Compton CC, et al., eds. American Joint Committee on Cancer Staging Manual. 7th ed. New York, NY: Springer; 2010:253.

15. Paquet N, Albert A, Foidart J, Hustinx R. Standardized uptake values in normal tissues. J Nucl Med. 2004;45:784-788.

16. Zasadny KR, Wahl RL. Standardized uptake values of normal-tissues at PET with 2-fluorine-L8-Fluoro-2-deoxy-D-glucose: variations with body weight and a method for correction. Radiology. 1993;189:847-850.

17. Vander Heiden MG, Cantley LC, Thompson CB. Understanding the Warburg effect: the metabolic requirements of cell proliferation. Science. 2009;324:10291033.

18. Younes M, Brown RW, Stephenson M, Gondo M, Cagle PT. Overexpression of Glut 1 and Glut 3 in stage I nonsmall cell lung carcinoma is associated with poor survival. Cancer. 1997;80:1046-1051.

19. Tanaka F, Yanagihara K, Otake Y, et al. Prognostic factors in resected pathologic (p-) stage IIIA-N2, non-small-cell lung cancer. Ann Surg Oncol. 2004;11:612618.

20. Thomas GV, Tran C, Mellinghoff IK, et al. Hypoxia-inducible factor determines sensitivity to inhibitors of mTOR in kidney cancer. Nat Med. 2006;12:122-127.

21. Hsu PP, Sabatini DM. Cancer cell metabolism: Warburg and beyond. Cell. 2008; 134:703-707.

22. Hoang JK, Hoagland LF, Coleman RE, Coan AD, Herndon JE 2nd, Patz EF Jr. Prognostic value of fluorine-18 fluorodeoxyglucose positron emission tomography imaging in patients with advanced-stage non-small-cell lung carcinoma. J Clin Oncol. 2008;26:1459-1464.

23. Vesselle H, Freeman JD, Wiens L, et al. Fluorodeoxyglucose uptake of primary non-small cell lung cancer at positron emission tomography: new contrary data on prognostic role. Clin Cancer Res. 2007;13:3255-3263.

24. Shafer D, Albain K. Lung cancer outcomes in women. Semin Oncol. 2009;36: 532-541.

25. Howlader N, Noone AM, Krapcho M, et al. SEER Cancer Statistics Review, 1975-2005. Bethesda, MD: National Cancer Institute. Available at: http:// seer.cancer.gov/statfacts/html/lungb.html, based on November 2010 SEER data submission, posted to the SEER website 2011. Accessed August 30, 2012.

26. Editorial. Taking sex into account in medicine. Lancet. 2011;378:1826.

27. Wei Q, Cheng L, Amos CI, et al. Repair of tobacco carcinogen-induced DNA adducts and lung cancer risk: a molecular epidemiologic study. J Natl Cancer Inst. 2000;92:1764-1772.

28. Donington JS, Colson YL. Sex and gender differences in non-small cell lung cancer. Semin Thorac Cardiovasc Surg. 2011;23:137-145.

29. Shigematsu H, Lin L, Takahashi T, et al. Clinical and biological features associated with epidermal growth factor gene mutations in lung cancers. JNCI. 2005;97:339-346.

30. Cook MB, McGlynn KA, Devesa SS, Freedman ND, Anderson W. Sex disparities in cancer mortality and survival. Cancer Epidemiol Biomarkers Prev. 2011;20:1629-1637.

31. Lally BE, Geiger AM, Urbanic JJ, et al. Trends in the outcomes for patients with limited stage small cell lung cancer: an analysis of the Surveillance, Epidemiology and End Results database. Lung Cancer. 2009;64:226-231.

32. Janssen-Heijnen ML, Houterman S, Lemmens VE, Brenner H, Steyerberg EW, Coebergh JW. Prognosis for long-term survivors of cancer. Ann Oncol. 2007; 18:1408-1413.

33. Callahan J, Kron T, Schneider-Kolsky M, Hicks RJ. Review: the clinical significance and management of lesion motion due to respiration during PET/CT scanning. Cancer Imaging. 2011;11:224-236. 